\title{
Hair Cell Toxicity in Anti-cancer Drugs: Evaluating an Anti- cancer Drug Library for Independent and Synergistic Toxic Effects on Hair Cells Using the Zebrafish Lateral Line
}

\author{
Yoshinobu Hirose ${ }^{1,2,3}$, Julian A. Simon ${ }^{4}$, and Henry C. Ou ${ }^{1,2,5}$ \\ ${ }^{1}$ Virginia Merrill Bloedel Hearing Research Center, University of Washington, Box 357923, Seattle, WA 98195-7293, USA \\ ${ }^{2}$ Department of Otolaryngology-Head and Neck Surgery, University of Washington, Seattle, WA 98195, USA \\ ${ }^{3}$ Department of Otolaryngology, Graduate School of Medicine, Yamaguchi University, Yamaguchi, Japan \\ ${ }^{4}$ Fred Hutchinson Cancer Research Center, Seattle, WA 98109, USA \\ ${ }^{5}$ Seattle Children's Hospital, Seattle, WA 98105, USA
}

Received: 6 April 2011; Accepted: 15 June 2011; Online publication: 6 July 2011

\begin{abstract}
Inner ear hair cell loss is the most common pathology seen after ototoxic drug injury. While certain drugs such as aminoglycosides and cisplatin are well-known to have dramatic ototoxic effects, it is probable that there are other drugs that cause occult degrees of hair cell loss and lesser degrees of hearing loss. Anti-cancer drugs are particularly strong candidates due to their general cytotoxicity. We have screened a library of 88 anti-cancer drugs (National Cancer Institute Approved Oncology Drugs Set) for drugs that damage hair cells of the zebrafish lateral line. The screen identified four out of five known ototoxic drugs. The screen also identified four out of seven suspected ototoxic drugs (drugs that have isolated case reports of patients developing hearing loss after administration). Five additional drugs with no known ototoxicity were identified as potentially novel ototoxins. Additional dose-response curves were performed to evaluate relative toxicity. Since anti-cancer drugs are often used clinically in combination, we also performed dose-response curves for a variety of anticancer drug combinations and demonstrated synergistic toxicity in five out of ten drug combinations that we tested. These findings support the use of the zebrafish lateral line as a screening tool to detect ototoxic effects in drugs and also suggest that ototoxicity should be

Correspondence to: Henry C. Ou · Virginia Merrill Bloedel Hearing Research Center · University of Washington · Box 357923, Seattle, WA 98195-7293, USA. Telephone: +1-206-6162598; fax: +1-206-2215685; email: henryou@u.washington.edu
\end{abstract}

considered in terms of drug regimens rather than individual drugs.

Keywords: zebrafish, hair cell death, anti-cancer drugs

\section{INTRODUCTION}

There is no standard screen for ototoxicity. When drugs are evaluated in clinical trials, attention is appropriately focused on larger organs (i.e., liver, kidney), rather than the tiny hair cells of the inner ear. Obviously, some drugs are found to induce hearing loss. These drugs are typically identified due to subjective patient complaints leading to more formalized investigation. However, for an ototoxic drug to garner enough attention, it must cause fairly consistent, significant auditory threshold shifts that are easily noticed by patients. Over the years, while many drugs are thought to be "potential ototoxins," there are very few well-established ototoxins outside of the aminoglycosides, loop diuretics, and platinum derivatives. It seems safe to assume, however, that these are only the most damaging ototoxins and that there are likely other drugs that cause lesser degrees of hearing loss that are not as easily detected.

Anti-cancer drugs, due to their general cytotoxicity and tissue penetration, are particularly likely to have ototoxic effects. However, it is important to note that 
hair cells of the inner ear are non-proliferating cells, and thus, the mechanism of hair cell injury may differ from the mechanism through which a drug causes other systemic toxicities (e.g., myelosuppression) or kills a cancer cell. A number of anti-cancer drugs, such as the platinum derivatives, nitrogen mustard, and vincristine, are known ototoxins (Cummings 1968; Stadnicki et al. 1975; Lugassy and Shapira 1990). A literature search of additional anti-cancer drugs reveals numerous drugs with isolated reports of patients developing hearing loss after treatment (Tibaldi et al. 1998; Moss et al. 1999; Saito et al. 2001; Attili et al. 2008). These case reports can be difficult to interpret because the patients are typically treated with multiple anti-cancer drugs, and thus, it is hard to attribute ototoxicity to a single drug or a combination of drugs. Phase I clinical trials for anticancer drugs designed to identify dose-limiting toxicities rarely if ever assess ototoxicity in heavily pre-treated cancer patients. While identifying ototoxic characteristics in anti-cancer drugs is unlikely to preclude usage, we have an obligation to the public to keep patients informed of the possible side effects of any drug used for treatment.

The zebrafish lateral line is a powerful system for identifying and evaluating drugs that damage mechanoreceptive hair cells, such as those in the inner ear of mammals (Ton and Parng 2005; Chiu et al. 2008). Due to the small size and optically clear body of larval zebrafish, hair cell damage can be rapidly assessed. In addition, due to the rapidity of experiments and high fecundity, zebrafish larvae are particularly valuable for thoroughly testing and modulating dose-response relationships. The zebrafish has been used effectively to examine hair cell toxicity and elucidate doseresponse relationships for aminoglycosides and cisplatin (Wiliams and Holder 2000; Harris et al. 2003; Ton and Parng 2005; Owens et al. 2009; Ou et al. 2007). In addition, it has been used to screen for toxicity to hair cells in a library of FDA-approved drugs and bioactives (Chiu et al. 2008).

We have used the zebrafish lateral line to screen a library of 88 anti-cancer drugs (National Cancer Institute Approved Oncology Drugs Set) for hair cell toxicity. This collection of FDA-approved drugs contains a representative sample of older cytotoxic drugs as well as new targeted therapies. To improve the sensitivity of our screen in comparison to our previous toxicity screens (Chiu et al. 2008), we screened the library at multiple drug concentrations and at multiple exposure durations. Screening in this fashion, we successfully identified four out of five known ototoxins and identified multiple other drugs as potential novel ototoxins. We further evaluated the anti-cancer drugs by testing common combinations of anti-cancer drugs and identified evidence of synergistic hair cell toxicity in five drug combinations.

\section{METHODS}

All zebrafish procedures described have been approved by the University of Washington Animal Care and Use Committee.

\section{Animals}

Zebrafish (Danio rerio) embryos of the AB wild-type strain were produced by paired matings of adult fish maintained at $28.5^{\circ} \mathrm{C}$ in the University of Washington zebrafish facility (Westerfield 2000). Embryos were maintained at a density of 50 embryos per $100 \mathrm{~mm}^{2}$ Petri dish in embryo media $(1 \mathrm{mM} \mathrm{MgSO} 4,120 \mu \mathrm{M}$ $\mathrm{KH}_{2} \mathrm{PO} 4,74 \mu \mathrm{M} \mathrm{Na}{ }_{2} \mathrm{HPO}_{4}, 1 \mathrm{mM} \mathrm{CaCl} 2,500 \mu \mathrm{M} \mathrm{KCl}$, $15 \mu \mathrm{M} \mathrm{NaCl}$, and $500 \mu \mathrm{M} \mathrm{NaHCO} 3$ in $\mathrm{dH}_{2} \mathrm{O}$ ). At 4 days post-fertilization (dpf), larvae were fed live Paramecia.

\section{Labeling}

For drug library screening, 5 dpf larval zebrafish were incubated with $2 \mu \mathrm{M}$ YOPRO-1 (Invitrogen) in embryo medium for $20 \mathrm{~min}$. Zebrafish were then rinsed three times in embryo medium. This protocol results in nuclear staining in the hair cells of the lateral line that is persistent for several hours.

\section{Library}

We screened a library of 88 anti-cancer drugs (National Cancer Institute Approved Oncology Drugs Set) available from the National Cancer Institute. The full listing of drugs tested is available from the National Cancer Institute (http://dtp.nci.nih.gov). The drugs are dissolved at $10 \mathrm{mM}$ concentration in DMSO.

\section{Screening}

We previously demonstrated that ototoxic drugs can require anywhere from $30 \mathrm{~min}$ (neomycin) to $6 \mathrm{~h}$ (cisplatin) to cause appreciable hair cell damage in the zebrafish lateral line. To account for this variability, the anti-cancer drug library was screened at multiple concentrations and durations. The general zebrafish screening protocol has been described previously (Chiu et al. 2008). Briefly, one YOPRO-1 labeled $5 \mathrm{dpf}$ zebrafish was placed into each well of a 96-well optical plate (NUNC) with in $150 \mu \mathrm{L}$ of embryo media. Drugs from the anti-cancer drug library were then added to the plate at $100 \mu \mathrm{M}$ concentration. Zebrafish larvae were exposed to the drug library for 1 or $6 \mathrm{~h}$ (screened separately) and then anesthetized with MS222 and imaged by fluorescence microscopy to determine whether hair cells 
were lost or showed evidence of damage. Each screen contained eight negative controls (1\% DMSO alone) and eight positive controls $(100 \mu \mathrm{M}$ neomycin $)$. Three outcomes were possible from the screen:

1. Hair cells undamaged, fish alive-this outcome suggested no damage and thus no further testing performed.

2. Hair cells damaged, fish alive-this outcome suggested the drug caused hair cell damage. A confirmatory retest under identical conditions was performed in triplicate. If the damage was replicated, dose-response testing was performed.

3. Hair cells undamaged or damaged, fish dead-In this outcome, the drug dose was reduced by one half and the test was repeated. If the same outcome (i.e., lethality) was observed, the dose was reduced further until reaching a dose at which the fish was alive, and hair cells could be assessed.

\section{Dose-response testing}

For drugs identified as potential ototoxic drugs from the initial screen and confirmatory retesting, doseresponse testing was performed. Five dpf zebrafish were exposed to the potential toxicant at $0,10,50$, 100, 200, and $400 \mu \mathrm{M}$ concentrations for 1 or $6 \mathrm{~h}$ (exposure duration matching duration used for screen that identified drug). Zebrafish were then fixed in $4 \%$ paraformaldehyde overnight at $4^{\circ} \mathrm{C}$ and then processed for immunohistochemistry and hair cell counts (see below).

\section{Combination drug testing}

To evaluate for possible synergistic effects, we tested common chemotherapeutic drug combinations that involved the "hits" identified by the screen. For combination drug testing, $5 \mathrm{dpf}$ zebrafish larvae were exposed to two drugs simultaneously for $6 \mathrm{~h}$. Zebrafish were then fixed in $4 \%$ paraformaldehyde overnight at $4^{\circ} \mathrm{C}$ and then processed for immunohistochemistry and hair cell counts. We purposely designed these combination regimens using relatively nontoxic individual doses of each drug, so that any synergistic effects would be easily identified.

\section{Hair cell counts/immunohistochemistry}

After fixation in $4 \%$ paraformaldehyde, zebrafish larvae were rinsed in phosphate-buffered saline (PBS) three times and then placed in blocking solution (1\% Triton-X, 5\% normal goat serum (NGS) in PBS) for 1 to $2 \mathrm{~h}$ at room temperature. Zebrafish were then incubated with anti-parvalbumin antibody (monoclonal, 1:400 in 1\% Triton-X, $1 \%$
NGS, in PBS) at $4^{\circ} \mathrm{C}$ overnight. Zebrafish were rinsed in $1 \%$ Triton-X in PBS (PBS-T) three times and then incubated in Alexa 488 goat anti-mouse fluorescent antibody (1:500, in $1 \%$ Triton-X, $1 \%$ NGS, in PBS) for $4 \mathrm{~h}$. Following secondary antibody labeling, zebrafish were rinsed in PBS-T followed by PBS and mounted between two coverslips in Fluoromount-G (Southern Biotech, Birmingham, AL, USA) for imaging. Mounted specimens were examined using a Zeiss Axioplan II microscope using a FITC filter set at a final magnification of $\times 200$ (Fig. 1). Hair cells from the SO1, SO2, O1, and OC1 neuromasts (Raible and Kruse 2000) were counted. Eight to 12 fish per dose were counted. Results were calculated as the mean hair cell survival as a percentage of the control (no drug).

\section{Statistics}

All values were calculated and presented as the mean value $\pm 1 \mathrm{SD}$. Statistical analyses were performed using one- and two-way ANOVA (VassarStats: http:/ / faculty. vassar.edu/lowry/VassarStats.html). Results were considered statistically significant if $p<0.05$. Combination indices for drug combinations were calculated using CompuSyn (ComboSyn, Inc. Paramus, NJ, USA). Effective dose 50\% (ED50) for dose-response curves was calculated using linear regression analysis using the least squares method with Microsoft Excel (Redmond, WA, USA). Note that linear regression models were found to fit the dose-response data better than non-linear models.

\section{RESULTS}

\section{Screening results}

As "proof of concept," we evaluated the ability of the zebrafish screening protocol to identify known and
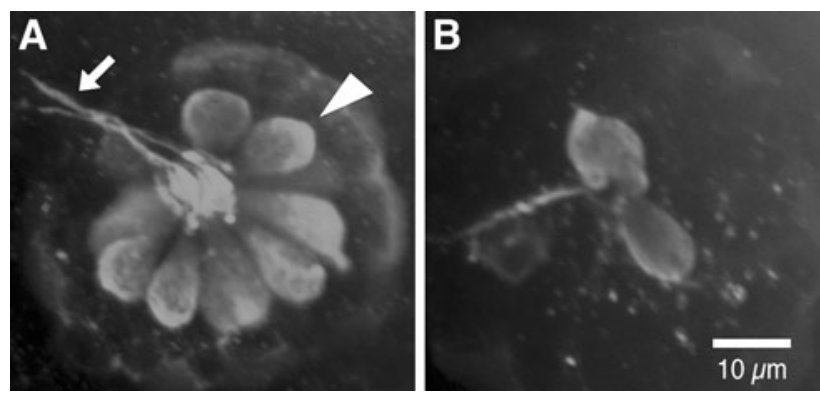

FIG. 1. Hair cells of the zebrafish lateral line. A Fluorescence microscopy of zebrafish lateral line neuromast with hair cell bodies labeled with anti-parvalbumin antibody. Triangle indicates a single hair cell within a characteristic rosette of hair cells. Arrow indicates kinocilia at apices of hair cells. Approximately nine hair cells are seen. B After treatment with cisplatin ( $200 \mu \mathrm{M}$ for $6 \mathrm{~h}$ ), extensive hair cell death occurs with only two hair cells remaining intact. Scale bar $=10 \mu \mathrm{m}$. 
potential ototoxic drugs. We performed a PubMed search of all drugs in the Approved Oncology Drugs set and divided the drugs into three categories: (1) known ototoxins-drugs that are well established to cause hearing loss in humans; (2) suspected ototoxins-drugs that have isolated case reports of hearing loss in human subjects after administration, or small animal studies; (3) no known ototoxicity-drugs with no published reports of hearing loss in humans and no significant animal studies. Based on the three categories listed above, there were five known ototoxins, seven suspected ototoxins, and 76 drugs with no known ototoxicity (Table 1 ). The screening protocol identified 13 out of the 88 drugs $(15 \%)$ as "hits." Of these drugs, four out of the five $(80 \%)$ known ototoxins and four out of seven (57\%) suspected ototoxins were among the hits. Five out of the $76(7 \%)$ remaining drugs with no known ototoxicity were identified as potential ototoxins. Drugs in the library that were not identified as toxic to hair cells are listed in Table 2.

\section{Carboplatin}

Of the known, well-established ototoxins, carboplatin was the only anti-cancer drug not detected by our screening protocol. We sought to evaluate whether this "miss" was due to a deficiency of the screening protocol or some unique quality of carboplatin ototoxicity. Carboplatin did not cause significant hair cell loss at up to $1 \mathrm{mM}$ concentration for $6 \mathrm{~h}$. This is in contrast to cisplatin, which is known to cause near complete hair cell loss at $1 \mathrm{mM}$ concentration after $6 \mathrm{~h}$ in the zebrafish lateral line (Ou et al. 2007).

\section{Dose-response functions}

We performed dose-response studies on the majority of the hits (Fig. 2; vinblastine, exemestane, and nitrogen mustard were excluded from further testing due to safety and availability issues). Treatment with all of the tested drugs led to dose-dependent hair cell loss in the zebrafish lateral line which was statistically significant by one-way ANOVA (Table 3). Raloxifene had the lowest ED50 of $12 \mu \mathrm{M}$, with hair cell survival decreasing from $100 \pm 10 \%$ to $60 \pm 11 \%$ after treatment with $10 \mu \mathrm{M}$ raloxifene (higher doses were not testable due to lethality to the fish; $p<0.01$, one-way ANOVA). The tyrosine kinase inhibitor sunitinib had an ED50 of $28 \mu \mathrm{M}$, with hair cell survival decreasing from $100 \pm$ $13 \%$ in the untreated control to $15 \pm 6 \%$ with $50 \mu \mathrm{M}$

TABLE 1

\begin{tabular}{|c|c|c|c|}
\hline \multicolumn{4}{|c|}{ Results from screen of $\mathrm{NCl}$ Approved Oncologic Drugs Set } \\
\hline & Mechanism of action & Screen used to ID ( $h$ ) & Reference \\
\hline \multicolumn{4}{|l|}{ Known ${ }^{\mathrm{a}}$ ototoxins identified } \\
\hline Cisplatin & DNA crosslinker & 1,6 & Stadnicki et al. (1975) \\
\hline Oxaliplatin & DNA crosslinker & 6 & Malhotra et al. (2010) \\
\hline Nitrogen mustard & Alkylating agent (mustard) & 6 & Cummings (1968) \\
\hline Vincristine & Microtubule inhibitor & 6 & $\begin{array}{l}\text { Lugassy and Shapira (1990); } \\
\text { Aydogdu et al. (2000) }\end{array}$ \\
\hline \multicolumn{4}{|l|}{ Known ${ }^{a}$ ototoxins missed } \\
\hline Carboplatin & DNA crosslinker & None & $\begin{array}{l}\text { Takeno et al. (1994); } \\
\text { Dean et al. (2008) }\end{array}$ \\
\hline \multicolumn{4}{|l|}{ Suspected ${ }^{b}$ ototoxins identified } \\
\hline Vinorelbine & Microtubule inhibitor & 1,6 & Tibaldi et al. (1998) \\
\hline Imatinib & Receptor tyr. kinase inhib & 1,6 & Attili et al. (2008) \\
\hline Doxorubicin & Topoisomerase poison & 1 & Saito et al. (2001) \\
\hline Vinblastine & Microtubule inhibitor & 6 & Moss et al. (1999) \\
\hline \multicolumn{4}{|l|}{ Suspected $^{\mathrm{b}}$ ototoxins missed } \\
\hline Erlotinib & Receptor tyr. kinase inhib & None & Koutras et al. (2008) \\
\hline Bleomycin & Induces DNA strand breaks & None & Dal et al. (1973) \\
\hline Lomustine & Alkylating agent (mustard) & None & Tsunoda (1970) \\
\hline \multicolumn{4}{|l|}{ Potential novel ${ }^{\mathrm{C}}$ ototoxins identified } \\
\hline Sunitinib & Receptor tyr kinase inhib. & 1 & None \\
\hline Raloxifene & Estrogen receptor mod & 6 & None \\
\hline Dactinomycin & Transcription inhibitor & 6 & None \\
\hline Carmustine & Alkylating agent & 6 & None \\
\hline Exemestane & Steroidal aromatase inhib & 6 & None \\
\hline
\end{tabular}

\footnotetext{
${ }^{a}$ Known defined as well established to cause hearing loss/hair cell death in humans

${ }^{\text {b }}$ Suspected defined as isolated case reports of hearing loss in humans, or limited animal data

${ }^{\mathrm{c}}$ Potential novel defined as having no reports in literature of ototoxicity
} 
TABLE 2

\begin{tabular}{|c|c|c|}
\hline \multicolumn{3}{|c|}{ Drugs not detected as toxic in hair cell toxicity screen of $\mathrm{NCl}$ Approved Oncologic Drugs Set } \\
\hline Allopurinol & Ixabepilone & Decitabine \\
\hline Busulfan & Ifosfamide & Nelarabine \\
\hline Thalidomide & Mitotane & Vorinostat \\
\hline Pentostatin & Megestrol acetate & Fulvestrant \\
\hline Tamoxifen citrate & Carboplatin & Anastrozole \\
\hline Rapamycin & Valrubicin & Letrozole \\
\hline Estramustine & Cyclophosphamide & Lenalidomide \\
\hline Fluorouracil & Paclitaxel & Clofarabine \\
\hline Floxuridine & Pemetrexed & Fludarabine \\
\hline Procarbazine & Methotrexate & Bortezomib \\
\hline Gemcitabine $\mathrm{HCl}$ & Plicamycin & Capecitabine \\
\hline Teniposide & Thiotepa & Celecoxib \\
\hline Bleomycin & Uracil mustard & Lapatinib \\
\hline Acrichine & Tretinoin & Mitoxantrone \\
\hline Hydroxyurea & Daunorubicin $\mathrm{HCl}$ & Gefitinib \\
\hline Methoxsalen & Topotecan $\mathrm{HCl}$ & Dasatinib \\
\hline Streptozocin & Dacarbazine & Sorafenib \\
\hline Chlorambucil & Temozolomide & Zolendronic acid \\
\hline Irinotecan $\mathrm{HCl}$ & Cytarabine $\mathrm{HCl}$ & Amifostine \\
\hline Nilotinib & Dexrazoxone & Melphalan \\
\hline Triethylenemelamine & Etoposide & Azacitidine \\
\hline Thioguanine & Docetaxel & Pipobroman \\
\hline Lomustine, CCNU & Arsenic trioxide & Aminolevulinic acid \\
\hline Cladribine & Imiquimod & Altretamine \\
\hline Mitomycin C & Erlotinib $\mathrm{HCl}$ & Mercaptopurine \\
\hline
\end{tabular}

sunitinib ( $p<0.01$, one-way ANOVA). In comparison, the ED50 for cisplatin was $129 \mu \mathrm{M}$, with hair cell survival decreasing from $100 \pm 6 \%$ in the untreated control to $26 \pm 3 \%$ after $200 \mu \mathrm{M}$ cisplatin. Doxorubicin demonstrated a similar degree of toxicity to cisplatin, with an ED50 of $139 \mu \mathrm{M}$, and hair cell survival decreasing to $37 \pm 5 \%$ after treatment with $200 \mu \mathrm{M}$ doxorubicin. The microtubule inhibitors, vincristine and vinorelbine, demonstrated less dramatic hair cell loss, with hair cell declining to $81 \pm 14 \%$ after treatment with $400 \mu \mathrm{M}$ vincristine $(\mathrm{ED} 50=1031 \mu \mathrm{M})$ and $72 \pm 8 \%$ after treatment with $400 \mu \mathrm{M}$ vinorelbine $(\mathrm{ED} 50=692 \mu \mathrm{M})$.

\section{Combinations}

Since anti-cancer drugs are generally given in combination, we investigated whether certain combinations of anti-cancer drugs (most involving cisplatin) demonstrated synergistic toxic effects on hair cells. Cisplatin combined with either vincristine, 5-fluorouracil, vinorelbine, doxorubicin, or etoposide and the combination of doxorubicin with vincristine are all commonly used chemotherapy drug combinations. In order to accentuate synergistic combination effects, we used relatively nontoxic concentrations of each individual toxicant. Ten different pairings of anti-cancer drugs were evaluated by dose-response analyses (Table 4). Five of the ten combinations demonstrated synergistic toxicity to hair cells. In particular, doxorubicin, vincristine, and vinorelbine demonstrated markedly increased hair cell toxicity when combined with a relatively nontoxic low dose of cisplatin ( $p<0.001$, two-way ANOVA; Fig. 3) with combination indices much less than 1 , indicating a high degree of synergistic toxicity (Chou and Talalay 1984). The imatinib/cisplatin and vincristine/doxorubicin combinations also exhibited synergistic toxicity, although to a much lesser extent. Other combinations tested, such as the common chemotherapeutic regimen of cisplatin with 5-fluorouracil, did not demonstrate any synergistic hair cell toxicity (Table 4 ).

\section{DISCUSSION}

Fine tuning the zebrafish hair cell toxicity screen

Since our initial screen for potential ototoxicity among FDA-approved drugs and bioactives (Chiu et al. 2008), we have worked toward improving the sensitivity of our drug screen. Our first toxicity screen was conducted at only 1-h exposure durations. Subsequent research has demonstrated that many wellestablished ototoxins such as gentamicin, kanamycin, and cisplatin (Ou et al. 2007; Owens et al. 2009) require longer time courses to damage hair cells of the zebrafish lateral line. To identify these types of ototoxins, we screened the anti-cancer drug library at 

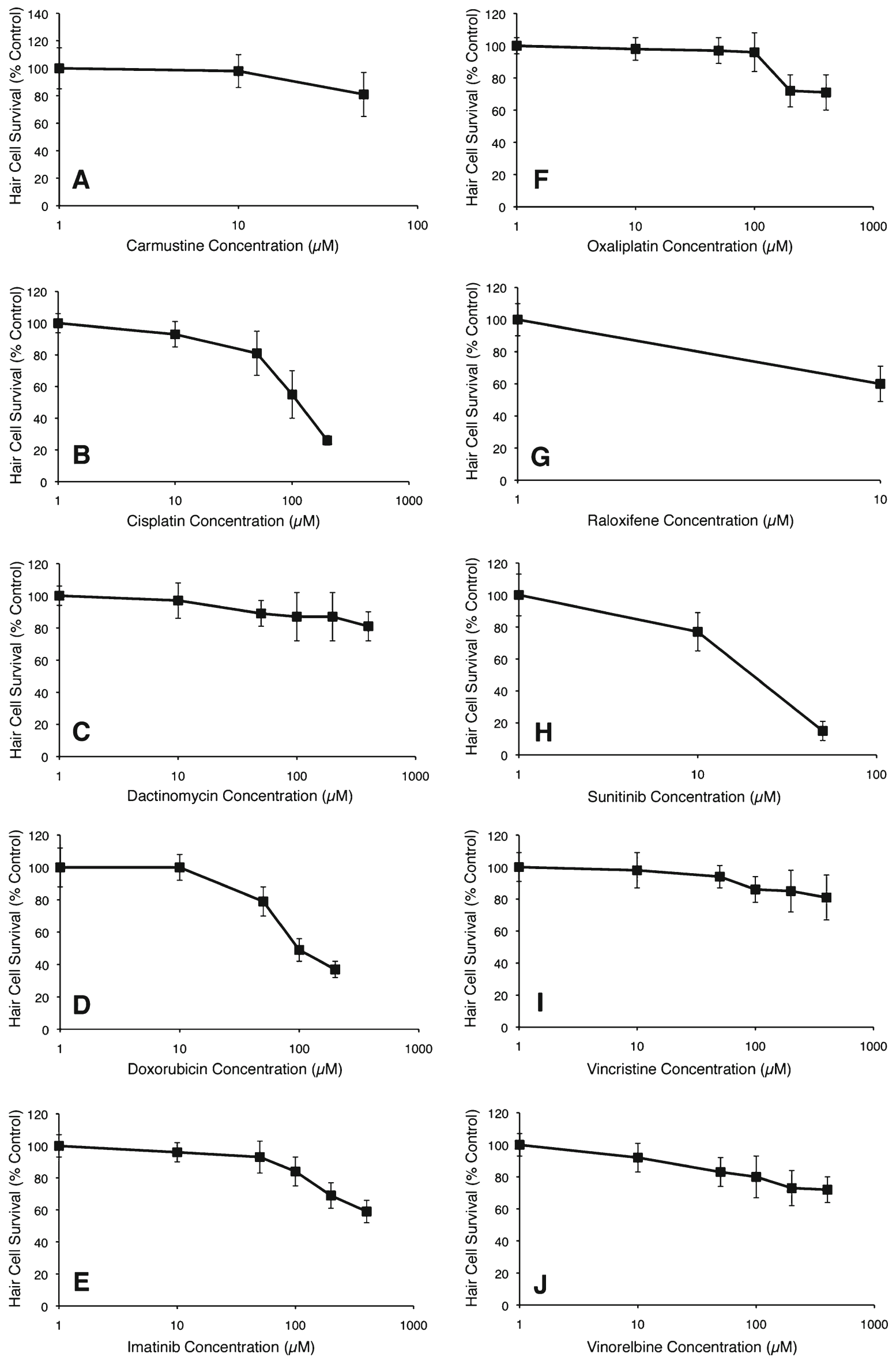
FIG. 2. Dose-response functions for anti-cancer drugs identified by toxicity screen. Dose-dependent hair cell loss was demonstrated for carmustine (A), cisplatin (B), dactinomycin (C), doxorubicin (D), imatinib $(\mathbf{E})$, oxaliplatin $(\mathbf{F})$, raloxifene $(\mathbf{G})$, sunitinib $(\mathbf{H})$, vincristine (I), and vinorelbine (J). Note that for some drugs, in particular carmustine, raloxifene, and sunitinib, fewer data points were obtained due to lethality to the fish at higher doses. Dose-dependent hair cell loss was statistically significant for all drugs $(p<0.05$, oneway ANOVA for carmustine and dactinomycin; $p<0.01$, one-way ANOVA for cisplatin, doxorubicin, imatinib, oxaliplatin, raloxifene, sunitinib, vincristine, and vinorelbine). All data points are the mean hair cell counts from seven to 10 fish. Error bars=SD.

both 1 and $6 \mathrm{~h}$ durations (independently). As expected, there were more hits (11) in the 6-h screen compared to the 1-h screen (five hits), with three drugs that were identified in both screens. It is notable, however, that the larger number of hits using the 6-h screen does not make the 1-h screen obsolete - some drugs are lethal to the zebrafish after $6 \mathrm{~h}$ and thus were identified as toxic to hair cells only in the 1$\mathrm{h}$ screen. That being said, the success of the 6-h screen demonstrated the importance of screening at longer durations in future studies.

It is important to note that the mechanism of toxicity for an individual toxin may vary depending on exposure duration. In fact, Owens et al. (2009) demonstrated that different damage protocols/durations for individual aminoglycosides appeared to cause hair cell loss through different pathways. Based on this, longer duration screens (e.g., 12- or 24-h screens) might identify additional toxic drugs that require longer durations to cause damage. However, in our experience, the inability of $5 \mathrm{dpf}$ zebrafish to survive for longer periods within 96-well plates limits the ability to test hair cell toxicity over longer durations in a toxicity screening protocol. Longer exposures can be done independent of a drug screen, as has been demonstrated effectively by Ton and Parng (2005), and certainly have an important role for evaluating candidate ototoxins.

\section{The zebrafish hair cell toxicity screen detects most but not all ototoxins}

Overall, the screen was successful at detecting four out of five known ototoxins and four out of seven suspected ototoxins. This rate of discovery is much higher than by chance (probability $<0.001$, chi-square analysis). It should be noted that most of the suspected ototoxins have only single case reports of hearing loss after administration of the drug, and in many cases, the anti-cancer drug was given in conjunction with other drugs. Thus, the three suspected ototoxins that were not detected may not be true "misses" since it is unknown whether they are truly ototoxic. Nevertheless, we were encouraged by the overall sensitivity of the screen to detect drugs that can affect hair cell survival. One should also note that in some cases, the screen identified drugs that only caused 20\% hair cell loss at high doses. This finding also speaks to the sensitivity of the screen to detect even drugs that cause small degrees of hair cell loss. For some of these less toxic drugs, the extrapolated ED50 (Table 3) was outside the range of the actual tested doses. In these cases, the dose required to cause $50 \%$ hair cell loss would in fact be lethal to the zebrafish and thus was not testable.

\section{Carboplatin}

Carboplatin was the one well-established ototoxin that was not detected by the screen, and interestingly, our additional testing of carboplatin failed to demonstrate any hair cell toxicity in the zebrafish, even at high

TABLE 3

Dose-response data showing hair cell survival with increasing doses of candidate ototoxins and ED50 values

\begin{tabular}{|c|c|c|c|c|c|c|c|c|}
\hline \multicolumn{9}{|c|}{ Hair cell survival (\% of control) } \\
\hline Drug & $O \mu M$ & $10 \mu \mathrm{M}$ & $50 \mu \mathrm{M}$ & $100 \mu \mathrm{M}$ & $200 \mu \mathrm{M}$ & $400 \mu \mathrm{M}$ & Duration (h) & ED50 $(\mu \mathrm{M})$ \\
\hline Carmustine* & $100 \pm 15$ & $98 \pm 12$ & $81 \pm 16$ & Dead & Dead & Dead & 6 & 131 \\
\hline Cisplatin** & $100 \pm 6$ & $93 \pm 8$ & $81 \pm 14$ & $55 \pm 15$ & $26 \pm 3$ & Dead & 6 & 129 \\
\hline Dactinomycin* & $100 \pm 6$ & $97 \pm 11$ & $89 \pm 8$ & $87 \pm 15$ & $87 \pm 15$ & $81 \pm 9$ & 6 & 1,130 \\
\hline Doxorubicin** & $100 \pm 12$ & $100 \pm 8$ & $79 \pm 9$ & $49 \pm 7$ & $37 \pm 5$ & Dead & 1 & 139 \\
\hline Imatinib** & $100 \pm 7$ & $96 \pm 6$ & $93 \pm 10$ & $84 \pm 9$ & $69 \pm 8$ & $59 \pm 7$ & 6 & 466 \\
\hline Oxaliplatin** & $100 \pm 5$ & $98 \pm 7$ & $97 \pm 8$ & $96 \pm 12$ & $72 \pm 10$ & $71 \pm 11$ & 6 & 615 \\
\hline Raloxifene** & $100 \pm 10$ & $60 \pm 11$ & Dead & Dead & Dead & Dead & 6 & 12 \\
\hline Sunitinib** & $100 \pm 13$ & $77 \pm 12$ & $15 \pm 6$ & Dead & Dead & Dead & 1 & 28 \\
\hline Vincristine** $^{* *}$ & $100 \pm 9$ & $98 \pm 11$ & $94 \pm 7$ & $86 \pm 8$ & $85 \pm 13$ & $81 \pm 14$ & 6 & 1,031 \\
\hline$V$ inorelbine** & $100 \pm 7$ & $92 \pm 9$ & $83 \pm 9$ & $80 \pm 13$ & $73 \pm 11$ & $72 \pm 8$ & 6 & 692 \\
\hline
\end{tabular}

Dead drug was lethal at this dose and hair cells could not be evaluated; ED50 (effective dose $50 \%$ ) calculated based on linear regression estimates

$* p<0.05$, one-way ANOVA; ${ }^{* *} p<0.01$, one-way ANOVA 
TABLE 4

Anti-cancer drug combinations and combination indices

\begin{tabular}{llll}
\hline Drug 1 & Drug 2 & Synergistic toxicity & Combination index \\
\hline Cisplatin & Vincristine & Yes & $0.03-0.05$ \\
Cisplatin & Vinorelbine & Yes & $0.08-0.28$ \\
Cisplatin & Doxorubicin & Yes & $0.12-1.05$ \\
Cisplatin & Imatinib & Yes & $0.56-1.09$ \\
Doxorubicin & Vincristine & Yes & $0.51-1.11$ \\
Cisplatin & Docetaxel & No & $0.72-1.27$ \\
Carboplatin & Vinorelbine & No & $0.95-3.26$ \\
Cisplatin & Etoposide & No & $1.15-1.87$ \\
Cisplatin & Carboplatin & No & $5.8-12.7$ \\
Cisplatin & 5-Fluorouracil & $\gg 10$ & \\
\hline
\end{tabular}

Note that for those combinations with combination indices $\gg 10$, suggesting antagonism, this is likely not true antagonism but a result of the minimal toxicity of the individual drug

doses. The reason for this is unclear and may be related to pharmacokinetic differences. However, one should note that the animal data on carboplatin ototoxicity is variable. Takeno et al. (1994) demonstrated selective loss of inner hair cells in carboplatin-treated chinchilla.
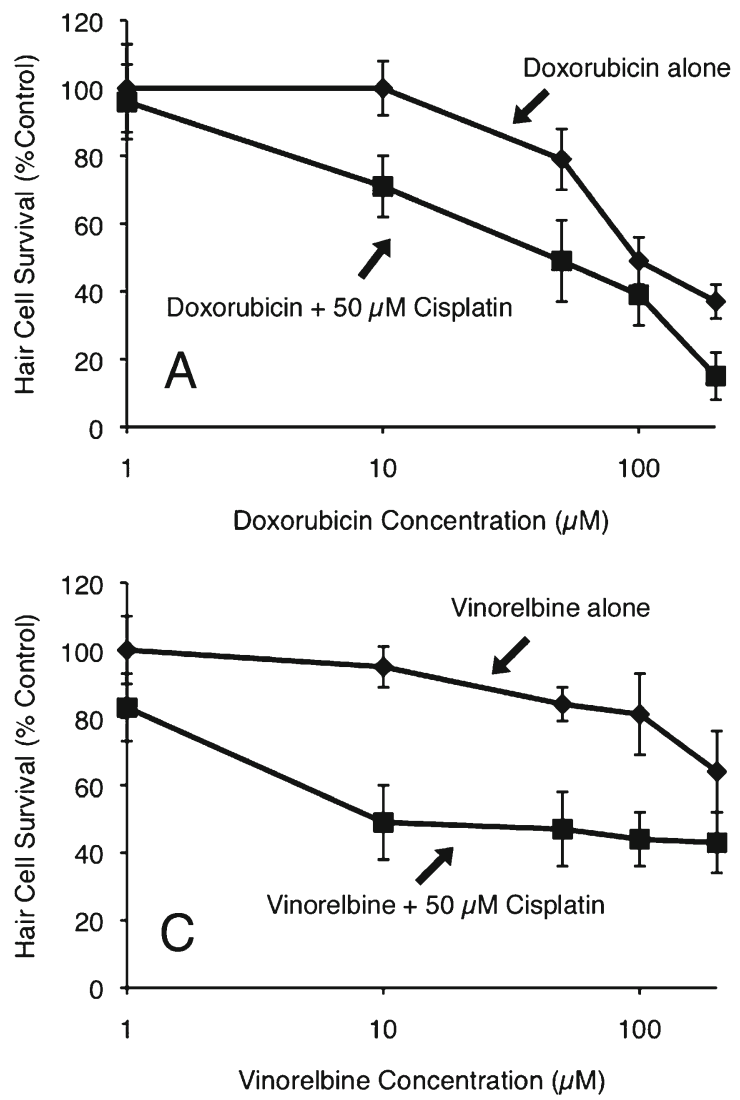

FIG. 3. Dose-response functions for anti-cancer drug combinations. A Dose-response curves for doxorubicin alone and doxorubicin with $50 \mu \mathrm{M}$ cisplatin. Adding a relatively nontoxic low dose of cisplatin results in a marked increase in doxorubicin toxicity, exceeding a simple additive effect (combination index $\ll 1$ ). B Dose-response curves for vincristine alone and vincristine with $50 \mu \mathrm{M}$ cisplatin. Vincristine alone had only a small amount of toxicity; however, addition of low dose cisplatin resulted in significant hair cell death (combination index $\ll 1$ ). C Dose-response functions for vinorelbine alone and vinorelbine with
Jiang et al. (2010) demonstrated hair cell damage in carboplatin-treated rat and chinchilla hair cells in vitro but required unusually high-dose exposure parameters (up to $5 \mathrm{mM}$ for $48 \mathrm{~h}$ ) to demonstrate hair cell loss. Furthermore, Dean et al. (2008) studied pediatric
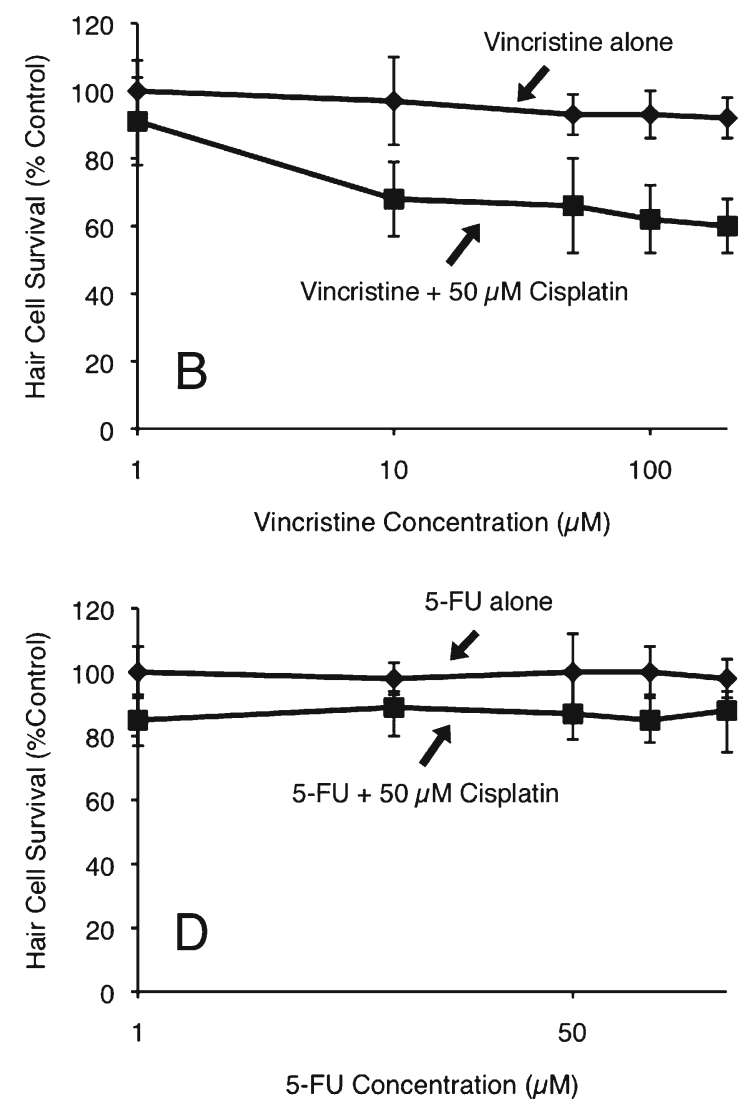

$50 \mu \mathrm{M}$ cisplatin. Vinorelbine alone demonstrates toxicity to hair cells. Addition of low dose cisplatin led to synergistic hair cell death (combination index «1). D Dose-response functions for 5 -fluorouracil (5-FU) alone and 5-FU with $50 \mu \mathrm{M}$ cisplatin. 5-FU alone was not toxic to hair cells, and addition of low dose cisplatin resulted in a minimal increase in hair cell death that was attributable to cisplatin alone. See Table 4 for combination indices for synergistic effects. All data points are the mean hair cell counts from seven to 10 fish. Error bars=SD. 
oncology patients receiving carboplatin-containing regimens and found that the incidence of hearing loss in children receiving carboplatin alone was $4 \%$, compared to $57 \%$ in children receiving cisplatin alone and $70 \%$ in children receiving both carboplatin and cisplatin. These findings are suggestive that carboplatin may have reduced ototoxicity compared to cisplatin, or that its ototoxicity may present primarily when combined with other anti-cancer drugs. We explored this possibility by testing for possible synergistic hair cell toxicity between cisplatin and carboplatin (Table 4) but found no synergistic interaction.

\section{Drug classes among toxins-expected versus unexpected}

Among the drug hits from the screen, a number of drug classes were identified (Table 1). As expected, cytotoxic drug classes such as platinum drugs, vinca alkaloids, and nitrogen mustard derivatives were identified as damaging to hair cells and have known mechanisms of action that would seemingly explain their ototoxicity. Other classes such as tyrosine kinase inhibitors and estrogen receptor blockers cause hair cell death through mechanisms that are unclear. These mechanisms of cell death could be explored by blocking the known pathways with which these drugs interact. However, it is well-known that drugs often have multiple targets, and as with other systemic toxicities, off-target activity may contribute to ototoxicity as well.

\section{Combination toxicity to hair cells}

Synergistic ototoxicity has been demonstrated previously between drugs such as loop diuretics and aminoglycosides (Santi et al. 1982) as well as drugs and noise (Li and Steyger 2009). Anti-cancer drugs are a particularly fertile ground for studying synergistic effects because these drugs are in general used in combinations of two to four drugs, and almost by definition often have some degree of cytotoxicity. We chose to utilize the theory and calculation of drug combination indices as described by Chou and Talalay (1984) as these concepts have not previously been applied to the study of synergistic hair cell toxicity. The combination index is broadly accepted as an effective method for analyzing drug combinations, particularly in the cancer literature. This index is calculated based on the median-effect equation and gives a quantitative number to define whether a drug combination is additive, synergistic, or antagonistic.

We found evidence of synergistic hair cell toxicity in five of the ten anti-cancer drug combinations. The combinations of cisplatin/vinorelbine, cisplatin/vincristine, and cisplatin/doxorubicin demonstrated significant synergistic toxicity to hair cells, with combination indices indicating strong synergism (combination index $\ll 1$; Chou 2006). With these combinations, we found that combining doses of the drugs that individually caused no hair cell loss could cause significant hair cell death when used together. This finding may have important clinical implications.

Our current findings suggest that when considering the ototoxic potential of an anti-cancer drug, one cannot simply consider an individual drug in isolation but rather the patient's entire medication profile. A single anti-cancer drug alone may not be ototoxic, but the combination with other anti-cancer drugs may provide an additional hair cell insult that leads to significant hearing loss. This finding may help us to better understand why some cancer patients will develop severe hearing loss during treatment while others develop none. This may also have implications for non-cancer patients, particularly geriatric patients who are more likely to be treated with multiple drugs.

Regarding the ototoxicity screen in general, while the detection of occult ototoxic effects is unlikely to preclude clinical usage, it is important to fully inform patients of any potential toxicity that they may experience as a result of medical therapy. In addition, one could imagine using a simple test such as the zebrafish screen described here to evaluate novel experimental drugs for potential ototoxic effects, such that during clinical trials patients can be monitored with appropriate audiologic testing.

\section{Caveats}

While hair cells of the zebrafish lateral line are anatomically and physiologically similar to inner ear hair cells, fish are not humans and thus findings in fish do not prove ototoxicity in humans. Mammalian in vitro testing is also inadequate to prove ototoxicity as it lacks the benefit of being in a living organism yet retains many of the same caveats as the zebrafish lateral line (no compartmentalization of fluid spaces and direct exposure of drug to hair cell). Mammalian in vivo testing is required to truly establish that a drug is ototoxic. It is possible that many of these drugs have insignificant inner ear penetration and thus may have no ototoxicity when used systemically. In addition, it is possible that electrolyte concentrations within the endolymph may alter the sensitivity of hair cells to certain ototoxins, as has been demonstrated by Coffin et al. (2009). Nevertheless, the effects of known ototoxins on the zebrafish lateral line hair cells, the recent growth of research validating the lateral line as a model for hair cell death, and the frequency of unexplained hearing loss during anti-cancer therapy are all suggestive that there may be similar findings in the mammal. Furthermore, many of these anti-cancer drugs are known to cross the blood-brain barrier and 
have good tissue penetration and thus have a higher likelihood of penetrating the inner ear. The versatility and low cost of the zebrafish lateral line screen for potential ototoxic side effects in drugs makes it possible to use this tool as a general screen for new agents and more importantly new combinations. Identification of potential ototoxicity could alert physicians to the possibility of hearing loss in their patients leading to more extensive audiology testing and prevention of permanent hearing loss.

\section{ACKNOWLEDGMENTS}

This research was supported by grants from NIH/NIDCD: K08DC009631, P30-DC004661, R01-DC009807, and R01DC005987-05. The authors would like to thank Drs. Edwin Rubel and David Raible for their input and Ms. Sarah Keating for her technical expertise.

\section{REFERENCES}

Attili VS, Bapsy PP, Anupama G, Lokanatha D (2008) Irreversible sensorineural hearing loss due to Imatinib. Leuk Res 32:991-992

Aydogdu I, Ozturan O, Kuku I, Kaya E, Sevinc A, Yildiz R (2000) Bilateral transient hearing loss associated with vincristine therapy: case report. J Chemother 12:530-532

Chiu LL, Cunningham LL, Raible DW, Rubel EW, Ou HC (2008) Using the zebrafish lateral line to screen for ototoxicity. J Assoc Res Otolaryngol 9:178-190

Chou TC (2006) Theoretical basis, experimental design, and computerized simulation of synergism and antagonism in drug combination studies. Pharmacol Rev 58:621-681

Chou TC, Talalay P (1984) Quantitative analysis of dose-effect RELATIONSHIPS: THE COMBINED EFFECTS OF MULTIPLE DRUGS OR ENZYME INHIBITORs. Adv Enzyme Regul 22:27-55

Coffin AB, Reinhart Ke, Owens KN, Raible DW, Rubel EW (2009) Extracellular divalent cations modulate aminoglycoside-induced hair cell death in the zebrafish lateral line. Hear Res 253(12):42-51

Cummings CW (1968) Experimental observations on the ototoxicity of nitrogen mustard. Laryngoscope 78:530-538

Dal I, Edsmyr F, Stahle J (1973) Bleomycin therapy and ototoxicity. Acta Otolaryngol 75:323-324

Dean JB, Hayashi SS, Albert CM, King AA, Karzon R, Hayashi RJ (2008) Hearing loss in pediatric oncology patients receiving carboplatincontaining regimens. J Pediatr Hematol Oncol 30:130-134

Harris JA, Cheng AG, Cunningham Ll, MacDonald G, Raible DW, RubEL EW (2003) Neomycin-induced hair cell death and rapid regeneration in the lateral line of zebrafish (Danio rerio). J Assoc Res Otolaryngol 4:219-234

Jiang H, Ding D, Fu Y, Salvi R (2010) Ototoxic effects of carboplatin in organotypic cultures in rats and chinchillas. Abstract of the Midwinter meeting of the Association for Research in Otolaryngology, Anaheim, CA

Koutras AK, Mastronikolis NS, Evans TR, Papadeas ES, Makatsoris T, KALOFONOS HP (2008) Irreversible ototoxicity associated with the use of erlotinib in a patient with pancreatic cancer. Acta Oncol 47:1171-1173

Li H, Steyger PS (2009) Synergistic ototoxicity due to noise exposure and aminoglycoside antibiotics. Noise Health 11:26-32

Lugassy G, Shapira A (1990) Sensorineural hearing loss associated WITH VINCRISTINE TREATMENT. Blut 61:320-321

Malhotra NK, Aslam R, Lipman SP, BiLSKI VJ (2010) Acute ototoxicity from a single infusion of oxaliplatin. Ear Nose Throat J 89:258261

Moss PE, Hickman S, Harrison BR (1999) Ototoxicity associated with vinblastine. Ann Pharmacother 33:423-425

Ou HC, Raible DW, Rubel EW (2007) Cisplatin-induced hair cell loss in zebrafish (Danio rerio) lateral line. Hear Res 233:46-53

Owens KN, Coffin AB, Hong LS, Bennett KO, Rubel EW, Raible DW (2009) Response of mechanosensory hair cells of the zebrafish lateral line to aminoglycosides reveals distinct cell death pathways. Hear Res 253:32-41

Raible DW, KRuse GJ (2000) Organization of the lateral line system in embryonic zebrafish. J Comp Neurol 421:189-198

Saito T, Zhang ZJ, Tokuriki M, Shibamori Y, Yamamoto T, Noda I, Oнtsubo T, SaIto H (2001) Doxorubicin ototoxicity is induced in mice by combination treatment with cyclosporin A. Acta Otolaryngol 121:787-793

Santi PA, Ruggero MA, Nelson DA, Turner CW (1982) Kanamycin and bumetanide ototoxicity: anatomical, physiological and behavioral correlates. Hear Res 7:261-279

Stadnicki SW, Fleischman RW, Schaeppi U, Merriam P (1975) Cisdichlorodiammineplatinum (II) (NSC-119875): hearing loss and other toxic effects in rhesus monkeys. Cancer Chemother Rep 59:467-480

Takeno S, Harrison RV, Mount RJ, Wake M, Harada Y (1994) Induction of selective inner hair cell damage by carboplatin. Scanning Microsc 8:97-106

Tibaldi C, Pazzagli I, Berrettini S, De Vito A (1998) A case of ototoxicity in a patient with metastatic carcinoma of the breast treated with paclitaxel and vinorelbine. Eur J Cancer 34:11331134

Ton C, PARng C (2005) The use of zebrafish for assessing ototoxic and otoprotective agents. Hear Res 208(1-2):79-88

Tsunoda Y (1970) Ototoxicity of nitrogen mustard-N-oxide (Nitromin). Nippon Jibiinkoka Gakkai Kaiho 73:581-598

Westerfield M (2000) The zebrafish book: a guide for the laboratory use of zebrafish (Danio rerio). University of Oregon Press, Eugene

Wiliams J, Holder N (2000) Cell turnover in neuromasts of zebrafish larvae. Hear Res 143(1-2):171-181 